CORRECTION

https://doi.org/10.1038/s41586-018-0069-3

\title{
Publisher Correction: Adolescence and the next generation
}

George C. Patton, Craig A. Olsson, Vegard Skirbekk, Richard Saffery, Mary E. Wlodek, Peter S. Azzopardi, Marcin Stonawski, Bruce Rasmussen, Elizabeth Spry, Kate Francis, Zulfiqar A. Bhutta, Nicholas J. Kassebaum, Ali H. Mokdad, Christopher J. L. Murray, Andrew M. Prentice, Nicola Reavley, Peter Sheehan, Kim Sweeny, Russell M. Viner \& Susan M. Sawyer

Correction to: Nature https://doi.org/10.1038/nature25759, published online 21 February 2018.

In Fig. $4 \mathrm{a}$ of this Analysis, owing to an error during the production process, the year in the header of the right column was '2016' rather than '2010'. In addition, in the HTML version of the Analysis, Table 1 was formatted incorrectly. These errors have been corrected online. 